\title{
PARTICLE DYNAMICS AND GAS-PHASE HYDRODYNAMICS IN A ROTATING CONE REACTOR
}

\author{
B. M. WAGENAAR, J. A. M. KUIPERS and W. P. M. VAN SWAAIJ \\ Department of Chemical Engineering, Twente University of Technology, PO Box 217, 7500 AE Enschede, \\ The Netherlands
}

(First received 16 June 1993; accepted in revised form 24 September 1993)

\begin{abstract}
The rotating cone reactor is a novel reactor type which can be used for rapid thermal solids processing. This paper focuses on particle dynamics and gas-phase hydrodynamics in a rotating cone reactor as a first stage to obtain a basic understanding of the processes that govern the performance of this reactor. Therefore, the flow of nearly spherical monosized PVC powder in a cold-flow rotating cone reactor has been studied under variation of the particle diameter $(140-780 \mu \mathrm{m})$, the cone rotational speed (up to $1800 \mathrm{rpm})$ and two different cone top-angles $\left(60\right.$ and $\left.90^{\circ}\right)$. The gas flow created by the rotating cone showed a marked influence on motion of particles smaller than $200 \mu \mathrm{m}$. A mathematical model is presented using a single-particle description, and a turbulent gas flow description near the wall according to the universal velocity profile. The experimentally observed residence time of the particles inside the present reactor is typically in the order of $0.2 \mathrm{~s}$.
\end{abstract}

\section{INTRODUCTION}

The rotating cone reactor is a novel reactor type which can be used for rapid thermal processing of solids. This paper focuses on particle dynamics and gas-phase hydrodynamics, to obtain understanding with respect to the relevant transport phenomena influencing the performance of the rotating cone reactor. A schematic representation of the rotating cone reactor geometry is presented in Fig. 1. Particles are introduced in the bottom of the rotating cone. The rotation of the cone induces particle motion and the particles are swept out of the reactor along a spiral path.

The rotating one reactor has a number of desirable (hydro-)dynamical features, among which the most important ones are:

(1) A short particle residence time, typically $0.2 \mathrm{~s}$. This short solid residence time is important in chemical systems in which the solids rapidly deactivate or rapidly react.

(2) Carrier gas is not required, which is advantageous in systems where product dilution with the carrier gas has to be avoided.

This work is limited to cold-flow experiments. In particular, the effect of operation conditions (i.e. cone rotational speed) and particle characteristics (i.e. particle diameter) on the particle residence time will be studied in detail from an experimental and theoretical point of view. Since accurate determination of the particle residence time involves the measurement of the local particle velocity along the particle trajectory, a short review of techniques which have been used by other investigators to measure the local particle velocity in gas-particle flows will be presented in the next section. Studies involving the transfer of heat to the particles and a reactor application will be a subject of further studies.

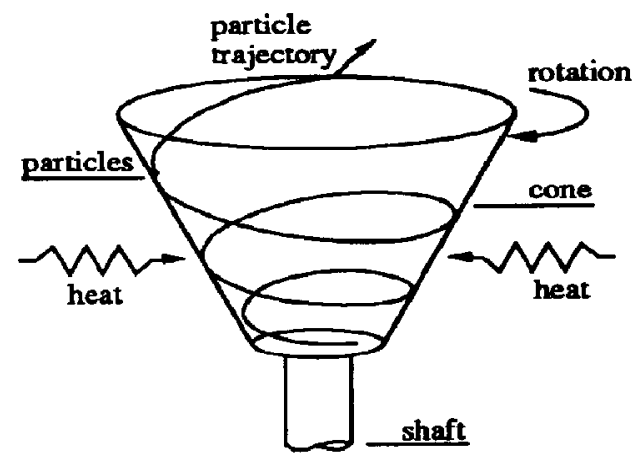

Fig. 1. Schematic representation of the rotating cone reactor.

\section{SELECTION OF THE PARTICLE VELOCITY MEASUREMENT METHOD}

A major characteristic of the rotating cone reactor is the residence time of particles in the reactor. This particle residence time can be derived from local particle velocity measurements by means of path integration. In the past decades several techniques have been employed to measure the local particle velocity. These techniques can broadly be divided into two classes:

(1) Point measurement techniques where the particle velocity is measured locally.

(2) Trajectory measurement techniques which are based on the measurement of the time lapse required for solid particles to travel the distance between the two points on a particle streamline.

\subsection{Impact probe}

An impact probe consists of a thin needle which is placed in the particle flow. This needle is connected to 
a stress-sensitive element, i.e. a piezocrystal that transforms the momentum of particle impact on the needle into an electrical signal; see Fig. 2. In a dilute particle flow, the particle collisions occur sequential and separated in time. The height, $h$, of recorded pulses is proportional to the momentum ( $m v)$ of the particle. This kind of particle flow measurement was studied by Raso and Tirabassa (1983). If individual particles cannot be distinguished, as is the case in a dense particle flow, the average pulse height, $h$, is proportional to the particle mass flow rate $(\rho v)$, and a relation between $\rho v$ and the pulse height $h$ as a function of the porosity has to be established. Heertjes et al. (1970) calibrated and operated an impact probe in the dense flow regime.

\subsection{Microwave radar technique}

The microwave radar technique has been used by Harris (1972) and Booth and Ridgway (1985) with an experimental set-up schematically represented in Fig. 3. The radar head consists of a Gunn diode which operates at a frequency of $10.7 \mathrm{GHz}$ and a power level of $10 \mathrm{~mW}$. The electromagnetic radar beam, when incident on moving particles, is scattered at a slightly different frequency. This frequency $f_{s}$ is related to the radar frequency $f_{i}$ by eq. $(2,1)$ :

$$
f_{s}=f_{i}\left(\frac{1+\frac{\mathbf{v}_{p} \cdot \mathbf{k}}{c}}{1+\frac{\mathbf{v}_{\mathbf{p}} \cdot \mathbf{l}}{c}}\right)
$$

where $c$ represents the speed of light. The scattered frequency from the particles interferes with a reference beam at the detector which results in a beat signal. The frequency of the beat signal follows from eq. (2.2):

$$
f_{D}=f_{s}-f_{i}=\frac{f_{i}}{c}(\mathbf{k}-1) \cdot \mathbf{v}_{p}
$$

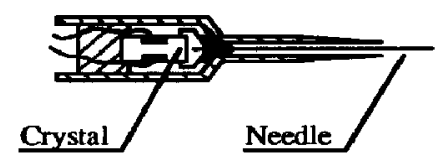

Fig. 2. The impact probe.

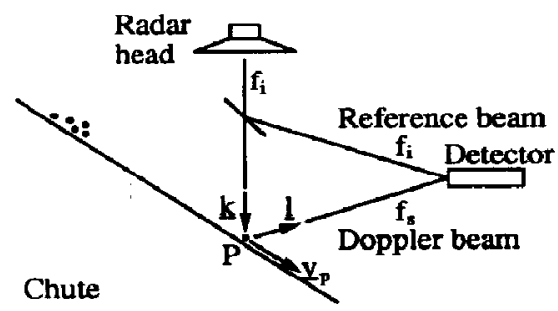

Fig. 3. Radar velocimetry.
The vectors $k$ and $\mathbf{I}$ are beam directional unit vectors which depend on the geometry of the experimental set-up. For particles moving at velocities between 0.1 and $5 \mathrm{~m} \mathrm{~s}^{-1}$, the Doppler frequency shift varies between 5 and $250 \mathrm{~Hz}$. As indicated by Booth and Ridgway (1985), the radar Doppler signal is produced as a result of microwave interactions throughout the thickness of the bed and, consequently, the measured velocities represent an average of all particles present in the flow field.

\subsection{Laser Doppler velocimetry}

There are basically two laser Doppler measurement techniques that differ in the choice of reference signal which interferes with the Doppler signal. If the reference beam originates from the laser source, the experimental set-up is equivalent to the radar set-up given in Fig. 3. Because of the identical physical nature of the laser Doppler set-up, the Doppler frequency shift can be calculated with eq. (2.2). An alternative way of generaling a reference beam is the use of the particle signal itself from a different angle. The corresponding experimental set-up is given in Fig. 4, showing a laser beam split into two beams which are focused on the particle. One of the beams can be regarded as the reference beam, and twofold application of eq. (2.2) yields the following expression for the differential Doppler shifts of the particle signal:

$$
f_{s 2}-f_{s 1}=\frac{f_{i}}{c}\left(\mathbf{k}_{2}-\mathbf{k}_{1}\right) \cdot \mathbf{v}_{p} \text {. }
$$

The advantage of the differential Doppler set-up is that the direction vector 1 has dropped out of eq. (2.3), which means that the detected Doppler shift is independent of the angular position of the detector relative to the particles. A much larger detector aperture can be used and, consequently, a larger signal can be detected with the differential Doppler set-up. Both systems are reviewed by Birchenough and Mason (1976) and Rosenberger (1975).

\subsection{Light reflection method}

A particle flow always contains non-uniformities like particle clusters, voids or even the particles themselves which reflect light differently. If the time-dependent reflection is measured on two positions along a particle streamline, both signals should be similar except from a shift of the time base, due to the travelling time of the signal between the two detectors. The measured time shift, obtained by autocorrelation, between the two signals is reciprocal to the particle speed. The light reflection technique has been used by Oki et al. (1977) and Hartge (1989).

One aspect which has to be considered in using this technique is the depth of light penetration in the solid suspension. In the case of the transmission of light through a randomly oriented collection of perfectly black particles, as schematically represented in Fig. 6 , the relationship between the light intensity and penetration depth can be derived from numerical simula- 


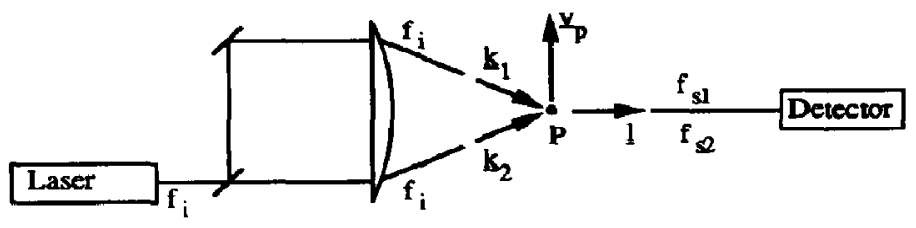

Fig. 4. Laser Doppler differential velocimetry.

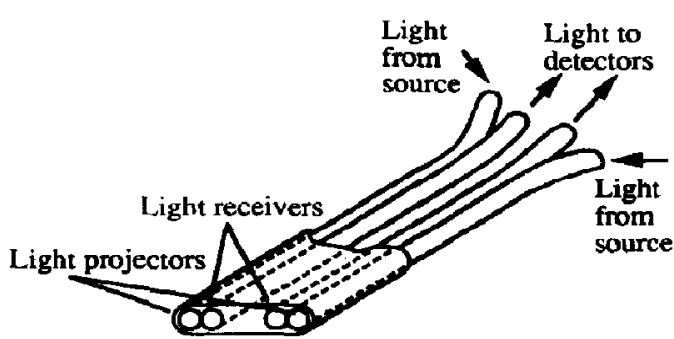

Fig. 5. Fibre optic probe.

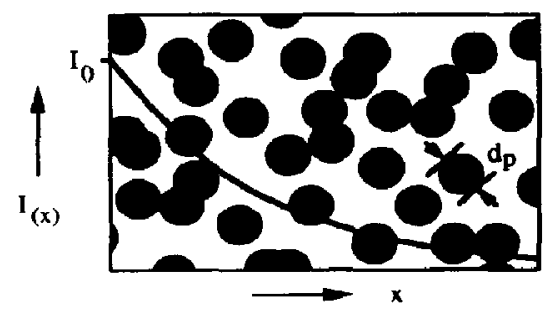

Fig. 6. Random configuration.

tions given by eq. (2.4):

$$
I=I_{\mathrm{o}} e^{-1.5\left[(1-2) / d_{p}\right] x} \text {. }
$$

On the basis of this exponential decay law it can be deduced that an increasing penetration depth results in an increasing measuring volume in more dilute suspensions.

\subsection{Photographic techniques}

On photographs the movement of a particle is visible as streaks (see Fig. 7) with a length proportional to the exposure time of the photograph. If a video camera is used with a variable shutter time, the photograph can be stored on magnetic tape, and can be analysed immediately. No calibration of this method is required, and the local particle velocity data are very detailed. The local particle velocity is the quotient between streak length and shutter time. This technique can only be used in an optically transparent flow, where eq. (2.4) can be used to estimate the transparency of the flow. The particle density in the rotating cone reactor was low enough to obtain an optically transparent flow. The photographic technique was chosen, to perform local particle velocity

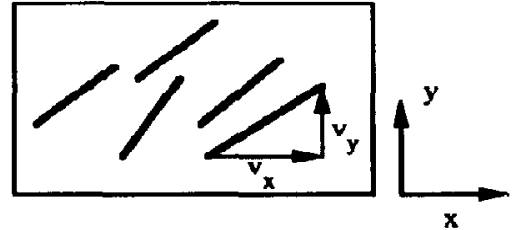

Fig. 7. Particle streaks.

measurements in the rotating cone reactor, because of the unambiguous visual measurements obtained with this technique. Other advantages of this technique are its relative low equipment costs and the fact that standard components can be used.

Table 1 contains the comparison between the different methods. Advantages are noted with a + sign, disadvantages or difficulties with a - sign.

\section{EQUIPMENT AND EXPERIMENTAL PROCEDURE}

In the present study the motion of the solid particles over the conical surface has been studied by a photographic technique. A high photographic contrast can be obtained by using a white powder on a black background, i.e. the conical surface. PVC was chosen as model solid because of the high light reflectivity of the PVC particles even down to the scale of a single particle. The density of this PVC powder is $1100 \mathrm{~kg} \mathrm{~m}^{-3}$, which is about twice the density of biomass.

The geometry of the cone used in this study is shown in Fig. 8. The experimental measurement setup is presented in Fig. 9. Due to the rotational motion of the cone, the particles are swept out of the reactor following a spiral path along the cone wall. An endoscope with camera has been used to record the particle motion. At a shutter-speed of $1 \mathrm{~ms}$, the particle movement can be seen as streaks of several $\mathbf{m m}$ length. This length is directly proportional to the local particle velocity. The local velocity of the gas phase has been measured with a hot wire anemometer, described by Lomas (1986).

\section{RESULTS}

The velocity of the particles has been measured at nine different heights in the cone. At each height the video recordings were analysed and the streaks were measured by length and angle. At the cone wall distance marks were placed and recorded in order to relate the measured streak length to its real physical 
Table 1. Comparison between different methods

\begin{tabular}{|c|c|c|c|c|}
\hline Method & $\begin{array}{l}\text { Calibration } \\
\text { required }\end{array}$ & $\begin{array}{c}\text { Non- } \\
\text { intrusiveness }\end{array}$ & $\begin{array}{c}\text { Optical } \\
\text { transparent } \\
\text { streams }\end{array}$ & $\begin{array}{c}\text { Optical } \\
\text { dense } \\
\text { streams }\end{array}$ \\
\hline Impact probe & - & - & + & + \\
\hline Microwave radar technique & - & + & + & + \\
\hline Laser Doppler velocimetry & + & + & + & - \\
\hline Light reflection method & - & - & + & + \\
\hline Photographic technique & + & + & + & - \\
\hline
\end{tabular}

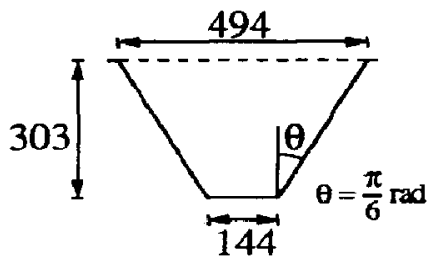

Fig. 8. The cone geometry. Sizes in $\mathrm{mm}$.

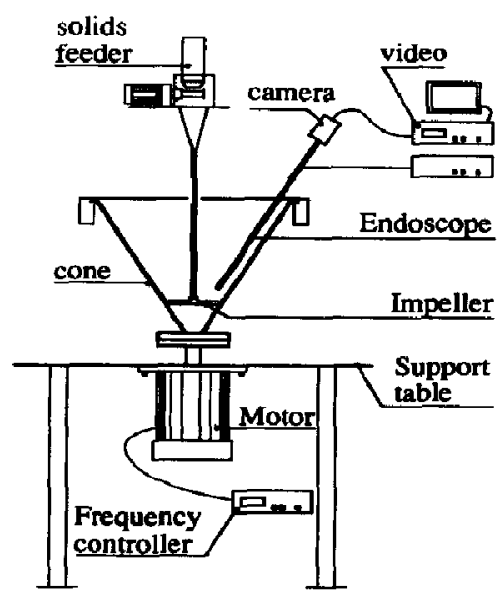

Fig. 9. The experimental set-up.

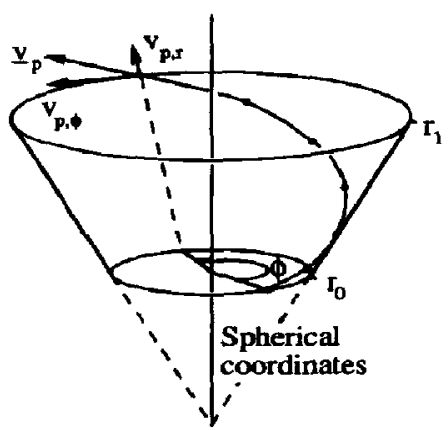

Fig. 10. The spherical coordinate system showing the velocity components and the angle $\phi$ of the particle trajectory. length. The streak angle was used for the decomposition of the particle velocity vector in its rotational and its radial component, respectively $v_{p, \phi}$ and $v_{p, r}$. The photographic technique used in the present study yields the projection of the particle trajectory on the conical surface which precludes the measurement of the velocity component normal to the cone wall. However, the velocity component normal to the cone wall is not relevant for determination of the particle residence time. At each cone height 30 streaks were measured and averaged. Figures 11 and 12 show, respectively, the measured radial velocity component $v_{r}$ and the angular velocity component $v_{\phi}$ as a function of the radius $r$.

The bars in Fig. 11 correspond to the variance in the measured local variable. From the experimental data like Figs 11 and 12, the particle residence time, the angle of the particle trajectory and the length of the particle trajectory can be calculated.

The measured local velocity possesses a fluctuating component due to an irregular particle shape and vibrations induced in the rotating equipment. The local particle velocity is approximately normal distributed as can be seen from Fig. 13. This figure is based on 2700 local particle velocity measurements.

The particle residence time in the rotating cone reactor can be calculated on the basis of the integral given in eq. (4.1).

$$
\tau=\int_{r_{0}}^{r_{1}} \frac{d r}{v_{p, r}}
$$

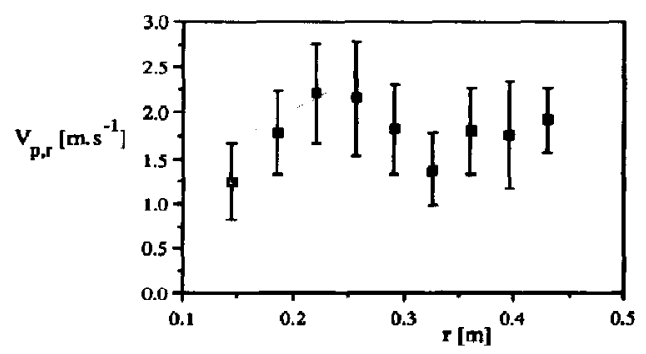

Fig. 11. The radial velocity component as a function of the radial coordinate. Particle diameter $140 \mu \mathrm{m}$, a cone rotation frequency of $13.2 \mathrm{~Hz}$ and a cone top-angle of $60^{\circ}$. 


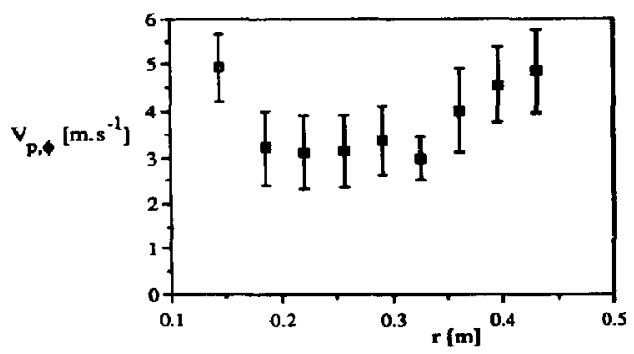

Fig. 12. The angular velocity component as a function of the radial coordinate. Particle diameter $140 \mu \mathrm{m}$ and a cone rotation frequency of $13.2 \mathrm{~Hz}$.

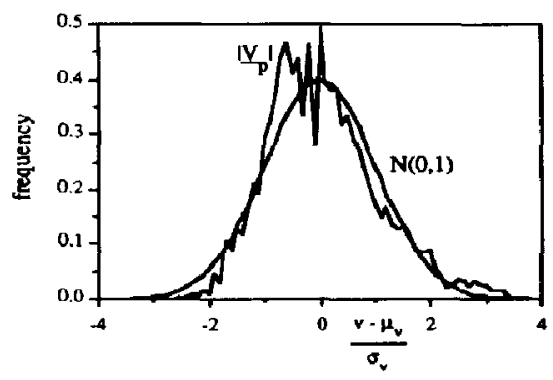

Fig. 13. The distribution of the local particle velocity.

The angle of the particle trajectory is defined as (see Fig. 10)

$$
\Delta \phi=\int_{r o}^{r_{1}} \frac{v_{p, \phi}}{r \sin \theta} \frac{\mathrm{d} r}{v_{p, r}} .
$$

The length of the particle trajectory is defined as

$$
\Delta s=\int_{r_{0}}^{r_{1}}\left|\mathbf{v}_{\mathrm{p}}\right| \frac{\mathrm{d} r}{v_{p r}}
$$

In order to obtain some qualitative information regarding the flow behaviour of the gas phase inside the rotating cone reactor, some preliminary experiments were performed in which the motion of injected smoke was studied with the aid of the above-mentioned photographic technique. In this way it was established that the rotational speed of the gas cloud in the core of the reactor was approximately $1 / 4$ of the cone rotational speed. These experiments also revealed the existence of a fully developed turbulent flow at cone rotational frequencies above $7.5 \mathrm{~Hz}$. Velocity measurements with a hot wire anemometer were in agreement with the qualitative smoke injection experiments. The results are shown in Fig. 14.

\section{THEORY}

\subsection{Single-particle model}

The mathematical model which governs the motion of the solid particles inside the rotating cone reactor consists of two parts: a force balance for the particle when it moves in free flight between two successive

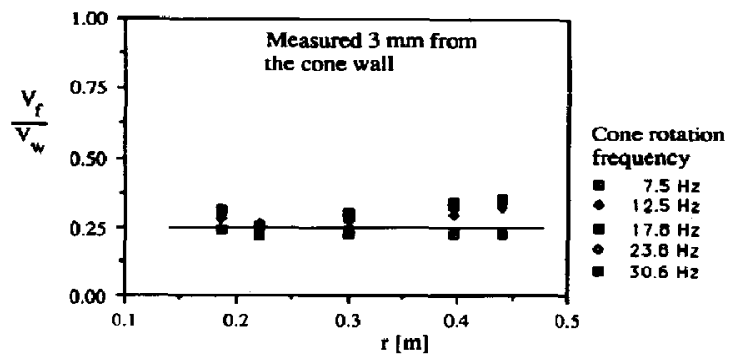

Fig. 14. The ratio between the gas-phase velocity and the wall velocity vs the radial coordinate.

wall collisions and a description of the collision between a particle and the cone wall. If a particle moves in free flight the only forces acting on the particle are the gravitational force and the drag force. The drag force causes coupling of the flow behaviour of the particles and the gas phase. The force balance for a particle reads as

$$
m_{p} \frac{\mathrm{d} \mathbf{v}_{p}}{\mathrm{~d} t}=\mathbf{F}_{d}+\mathbf{F}_{q}
$$

where $F_{d}$ represents the drag force and $F_{g}$ the gravitational force. The position vector of the particle is related to its velocity by

$$
\frac{\mathrm{d} \mathbf{X}_{p}}{\mathrm{~d} t}=\mathbf{v}_{p}
$$

The gravitation and drag force can be expressed, respectively, as

$$
\begin{gathered}
F_{g}=m_{p} g \\
F_{d}=C_{d} \frac{\pi}{4} d_{p}^{2} \frac{1}{2} \rho_{\mathrm{f}}\left|\mathbf{v}_{f}-\mathbf{v}_{p}\right|\left(\mathbf{v}_{f}-\mathbf{v}_{p}\right) .
\end{gathered}
$$

Particles moving over a surface in cases where the surface occupation is less than $1 \%$ usually show a bouncing behaviour, especially at the high particle speeds as encountered in the rotating cone reactor. Tsuji et al. (1989) numerically simulated the bouncing behaviour on a smooth wall of particles that deviate less than 3\% from a spherical shape. Even this small deviation from sphericity caused a scatter in particle velocities of $15 \%$ after impact with a wall. The bouncing behaviour of the particles has been taken into account by introduction of the deflection angle $\beta$, which is the difference between the incident and the reflected angle of a bouncing particle. The particle velocity after collision with the wall is given by eq. (5.5) (see Fig. 15):

$$
\begin{aligned}
\mathbf{v}_{p, 2}= & \left(\cos \beta-\frac{\left|\mathbf{m n} \cdot \mathbf{v}_{p, 1}\right|}{\left|(\mathbf{l}-\mathbf{n n}) \cdot \mathbf{v}_{p, 1}\right|} \sin \beta\right)(1-\mathbf{n n}) \cdot \mathbf{v}_{p, 1} \\
& -\left(\cos \beta+\frac{\left|(\mathbf{l}-\mathbf{n n}) \cdot \mathbf{v}_{p, 1}\right|}{\left|\mathbf{n n} \cdot \mathbf{v}_{p, 1}\right|} \sin \beta\right) \mathbf{n n} \cdot \mathbf{v}_{p, 1} .
\end{aligned}
$$




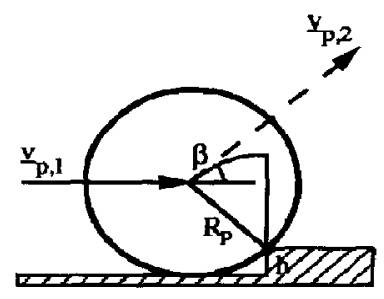

Fig. 15. A bouncing particle.

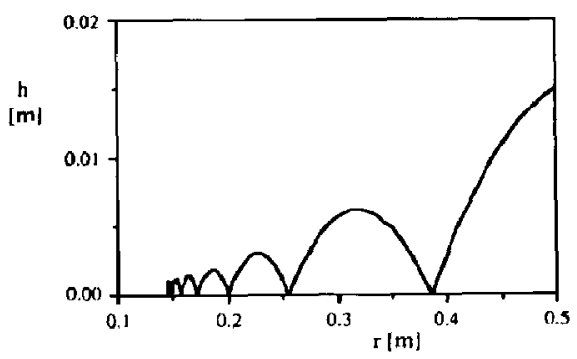

Fig. 16. The height of a bouncing particle as a function of its position in the rotating cone. Particle diameter $780 \mu \mathrm{m}$, cone rotation frequency $13.2 \mathrm{~Hz}$, a cone top-angle of $60^{\circ}$ and a roughness height $h$ equal to $0.1 \mu \mathrm{m}$.

As is evident from Fig. 15, particle deflection with conservation of momentum occurs if a particle bounces on a surface with roughness $h$. The difference between the incident and reflected angle is denoted by $\beta$, a parameter which is important for the description of the collision process. This coefficient is of phenomenological nature and incorporates the surface roughness, the particle shape factor and vibrations of the rotating surface. The surface roughness $h$ can be related to the deflection angle $\beta$ by

$$
\cos (\beta)=1-\frac{h}{R_{p}}
$$

An example of the results of a numerical simulation is presented in Fig. 16 which shows the distance of the particle from the cone wall as a function of the radial coordinate.

The bouncing height distribution of the particles has been measured by collecting bouncing particles at different heights from the cone wall in a small tube which has been inserted in the solids flow. This tube was positioned with its axis parallel to the solids streamlines. The solids collector was placed at the reactor exit and did not disturb the solids flow inside the reactor. Based on the particle bouncing heights observed in our particular reactor, which vary from 5 to $20 \mathrm{~mm}$, a roughness length $h$ of $0.1 \mu \mathrm{m}$ has been chosen.

The bouncing height at the reactor exit has also been calculated from the theoretical model for different particle diameters $(140-780 \mu \mathrm{m})$ and cone rota- tional frequencies $(5-30 \mathrm{~Hz})$. Values of these calculated bouncing heights vary between 13 and $17 \mathrm{~mm}$ and agree qualitatively with the experimentally determined bouncing heights at the reactor exit (see Fig. 16).

The volumetric solids fraction in the suspension on the cone wall has a value of $4 \times 10^{-5}$ for a typical experiment (solid mass flow rate $1 \mathrm{~g} / \mathrm{s}$, bouncing height $15 \mathrm{~mm}$ and a solid residence time $0.2 \mathrm{~s}$.). This fraction is sufficiently low to permit the use of a singleparticle model to describe the solids flow.

The trajectory of the particles originates at the position where the particles leave the impeller mounted in the base of the cone. The initial values which are required for the computation of the particle trajectories are given by

$$
\begin{aligned}
R_{0} & =0.071 \mathrm{~m} \\
v_{p, r} & =0 \\
v_{p, \phi} & =\omega_{\text {cone }} R_{0} .
\end{aligned}
$$

\subsection{Gas-phase description}

With respect to the flow behaviour of the gas phase inside the rotating cone reactor two distinct regions can be identified, each with different hydrodynamic behaviour: a gas cloud in the centre of the cone rotating at an uniform angular speed, and turbulent boundary layers in the vicinity of the cone wall. Gasphase velocities inside the gas cloud have been obtained by hot wire anemometry and are depicted in Fig. 14. For the description of the velocity profiles in the turbulent boundary layers the well-known universal velocity profile has been used. When applying the universal velocity profile, described by Schlichting (1960), two other layers have to be considered, the logarithmic layer and the viscous sublayer. The eccentricity of the cone causes no separation of the boundary layer. This behaviour can be expected on the basis of the experiments of Jacobs (1989), who studied turbulent flows over wavy surfaces. (The deviation in circularity of the cone's cross-section is $1 \mathrm{~mm}$.)

The velocity profile of the gas phase is given by

$$
\mathbf{v}_{f}=v_{f} \mathbf{e}_{\phi}
$$

If the nomenclature of the universal velocity profile is used, the gas-phase velocity $\nabla_{f}$ is a function of the dimensionless distance to the cone wall $y^{+}$and the dimensionless shear velocity acting on the cone wall $u^{*}$. The parameter $\delta$ measures the distance from the cone wall where the macroscopic gas flow changes in the boundary layer flow:

$$
y^{+}=\left(R_{w}-R\right) \frac{u^{*}}{v}
$$

and

$$
u^{*}=\frac{1}{4} \omega k \delta
$$


The profile of the angular gas-phase velocity consists of three parts. The first part is derived from the experiments given in Fig. 14 and describes the angular gas-phase velocity profile in the turbulent core of the rotating cone. Equations (5.14) and (5.15) give the velocity profile in, respectively, the logarithmic layer and the viscous sublayer.

$$
\begin{gathered}
v_{f}=\frac{1}{4} \omega R, \quad y^{+}>\delta \frac{u^{*}}{v} \\
v_{f}=\omega R_{w}-\frac{1}{k} u^{*}\left(2+\ln y^{+}\right), \quad 11<y^{+}<\delta \frac{u^{*}}{v} \\
v_{f}=\omega R_{w}-u^{*} y^{+}, \quad y^{+}<11
\end{gathered}
$$

The parameter $\delta$ couples the macroscopic flow described by eq. (5.13) with the flow in the turbulent boundary layer equation (5.14), and is therefore given by

$$
\frac{1}{3} \delta\left\{1+\ln \left(\frac{\omega k \delta^{2}}{4 v}\right)\right\}=R_{w}
$$

A more accurate description of the flow behaviour of the gas phase can be obtained if a computational fluid dynamics (CFD) model is applied. Equation (5.13) could then be obtained a priori from such simulations. This approach has not been used in this study because it was possible to obtain eq. (5.13) from the experiments given in Fig. 14

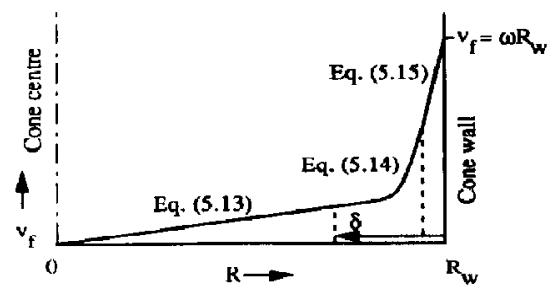

Fig. 17. Assumed radial profile of the angular gas velocity.

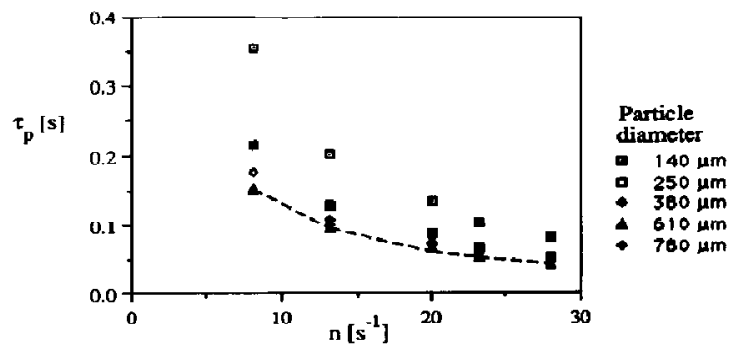

Fig. 18. The experimentally determined particle residence time as a function of the cone rotation frequency and the particle diameter. The cone geometry was a cone top-angle of $60^{\circ}$ and a cone height of $303 \mathrm{~mm}$. Solid mass flow rate: $1 \mathrm{~g} / \mathrm{s}$.

\section{MODEL VERIFICATION}

\subsection{Measurements with the $60^{\circ}$ top-angle cone}

Particle velocity measurements have been carried out for various particle diameters and cone rotational speeds. From these measurements, the residence time of the particles vs the cone rotational speed has been determined by evaluating the integral on the righthand side of eq. (4.1). The results are presented in Fig. 18. As expected, the particle residence time decreases rapidly with increasing cone rotational frequencies because of increased particle velocities. Furthermore, the results shown in this figure indicate that the viscous force becomes important relative to the particle mass inertia, for particles smaller than $200 \mu \mathrm{m}$. Particles larger than $400 \mu \mathrm{m}$ seem to be unaffected by the viscous forces and, consequently, the residence time of particles with increasing diameter approaches an asymptotic solution given by the dashed curve in Fig. 18.

Figure 19 shows a parity plot of the theoretically calculated particle residence time vs the experimentally determined residence time. As is evident from Fig 19 the agreement between the model predictions and the experimental results is reasonable.

The measured radial and angular particle velocity components can be used to evaluate the integral on the right-hand side of eq. (4.2), yielding the trajectory angle $\phi$ covered by the particles (see Fig. 10). The results of the calculations are presented in Fig. 20 . As is evident from this figure particles smaller than $200 \mu \mathrm{m}$ are strongly influenced by viscous forces. The trajectory angle of particles with a larger diameter approaches an asymptotic solution which is represented by the dashed curve in Fig. 20. This asymptotic solution of particles larger than $400 \mu \mathrm{m}$ shows a weak dependence of the cone rotational speed, which indicates that the shape of the trajectories of the larger particles is independent of the cone rotational speed.

If the model calculations and the experimental results are compared, the maximum deviation between model and experiment is less then $30 \%$ as can be seen from Fig. 21. In judging the accuracy of the model predictions it should be borne in mind that the present model does not employ any fitted parameters; all of them have been obtained from established empirism.

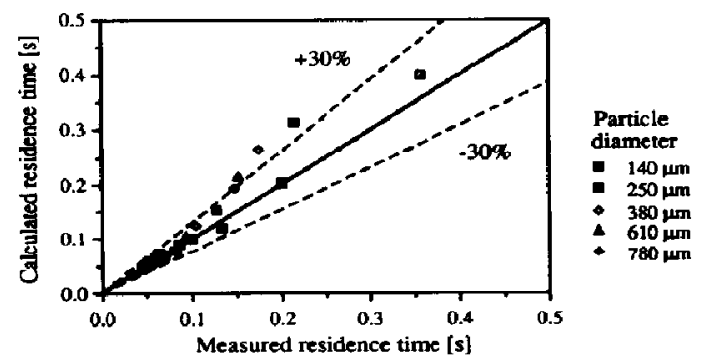

Fig. 19. Parity plot showing the theoretically calculated vs the experimentally determined particle residence time. 


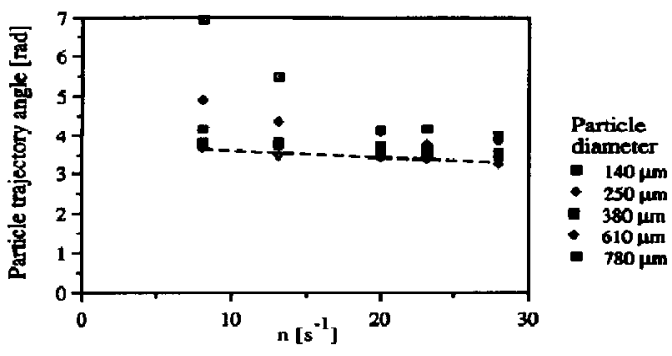

Fig. 20. Experimentally determined particle trajectory angle as a function of the cone rotation frequency and the particle diameter. Cone top-angle $60^{\circ}$ and a cone height of $303 \mathrm{~mm}$. Solids mass flow rate: $1 \mathrm{~g} / \mathrm{s}$.

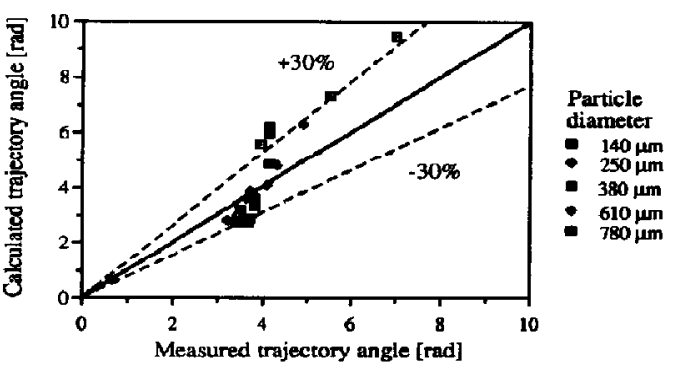

Fig. 21. Parity plot showing the calculated particle trajectory angle vs the measured trajectory angle.

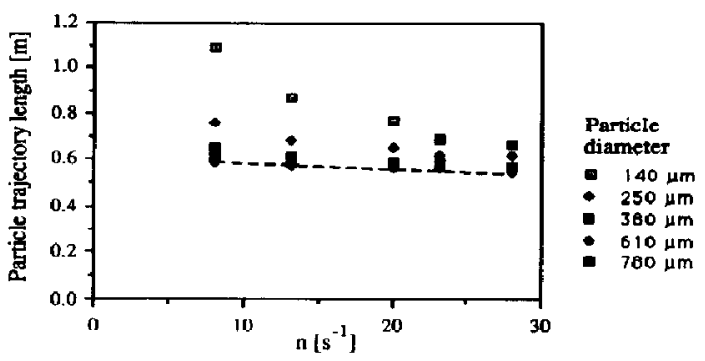

Fig. 22. The experimentally determined particle trajectory length as a function of the cone rotation frequency and the particle diameter. Cone top-angle $60^{\circ}$ and a cone height of $303 \mathrm{~mm}$. Solids mass flow rate: $1 \mathrm{~g} / \mathrm{s}$.

The length of a particle trajectory can be obtained by evaluating the integral on the right-hand side of eq. (4.3), using the measured particle velocity components. For particles larger than $500 \mu \mathrm{m}$ the viscous force is small compared to the mass inertia of the particles and as a consequence an asymptotic solution is reached which holds for particles larger than $400 \mu \mathrm{m}$. The asymptotic solution is given by the dashed curve in Fig. 22. Again the small dependence of the length of the particle trajectories on the cone rotational speed indicates that the shape of the trajectories is independent of the cone rotational speed.
If the model calculations and the experimental results are compared, the maximum deviation between model and experiment is less then $30 \%$, as can be seen from the parity plot shown in Fig. 23.

\subsection{Measurements with the $90^{\circ}$ top-angle cone}

In addition, measurements were performed in a cone with a top-angle of $\pi / 2$ radians as schematically represented in Fig. 24. The gas-phase velocity in the vicinity of the cone wall was measured with a hot wire anemometer. The results of these measurements are shown in Fig. 25. The angular gas-phase velocity in the turbulent core of the rotating cone as described by

$$
v_{s}=\frac{1}{3} \omega R
$$

is included in this figure. This velocity profile was also used in the numerical simulations duplicating the experiments. The multiplication factor used in eq. (6.1) differs from the multiplication factor used in eq. (5.13). Due to the rotation of the cone possibly a secondary gas flow can be induced which is directed along the

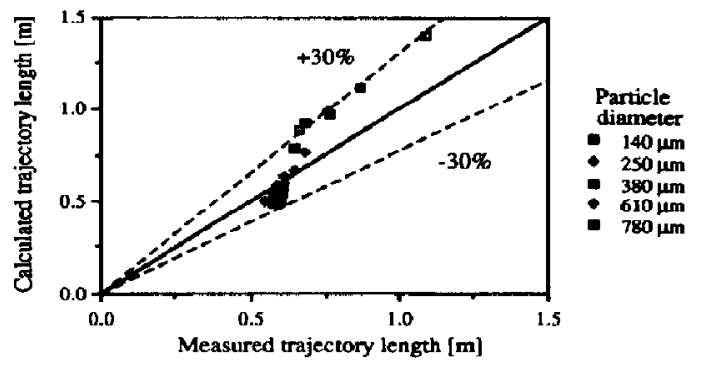

Fig. 23. Parity plot showing the calculated particle trajectory length vs the measured trajectory length.

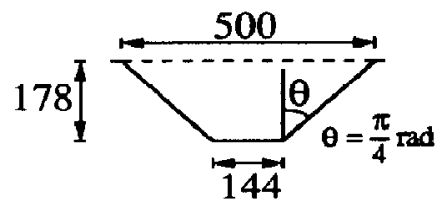

Fig. 24. Schematic representation of the cone geometry.

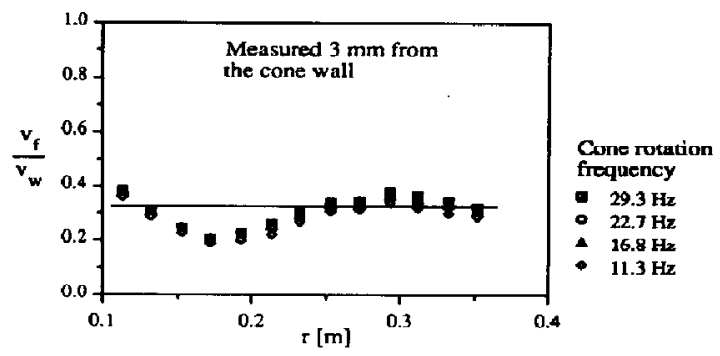

Fig. 25. The angular gas-phase velocity vs the radial coordinate. 
cone wall from the bottom of the cone towards the cone exit (radial direction). It returns to the cone bottom along the rotation centre of the cone. However, the hot wire anemometer (which is also sensitive for a radial velocity component of the gas phase) did not indicate a significant secondary gas flow radial velocity component because the local gas-phase velocity measured by the anemometer agreed quite well with the angular gas-phase velocity obtained by the smoke experiments. In addition, the model which does not incorporate a secondary gas flow along the cone wall in radial direction predicts particle residence times, trajectory lengths and angles which agree well with the experimental results. Therefore, the secondary gas flow, if present, is not large enough to influence the particle residence time (etc.) within the experimental error.

Similar to the procedure described in Section 6.1, the particle residence time $\tau_{p}$ in the rotating cone reactor can be obtained from the experimentally determined components of the local particle velocity and eq. (4.1). The results of these calculations are presented in Fig. 26, showing the particle residence time as a function of the cone rotation frequency and the particle diameter. The dashed curve in this figure represents the asymptotic solution for large particles where the gas phase has a negligible influence on the particle residence time.

On the basis of the theoretical model presented in Section 5 , the corresponding theoretically calculated particle residence times can be plotted against the

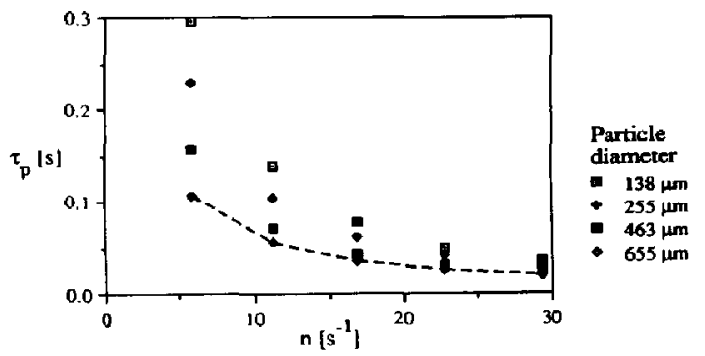

Fig. 26. Experimentally determined particle residence times as a function of the cone rotation frequency and the particle diameter. Cone top-angle $90^{\circ}$ and a cone height of $178 \mathrm{~mm}$. Solids mass flow rate: $1 \mathrm{~g} / \mathrm{s}$.

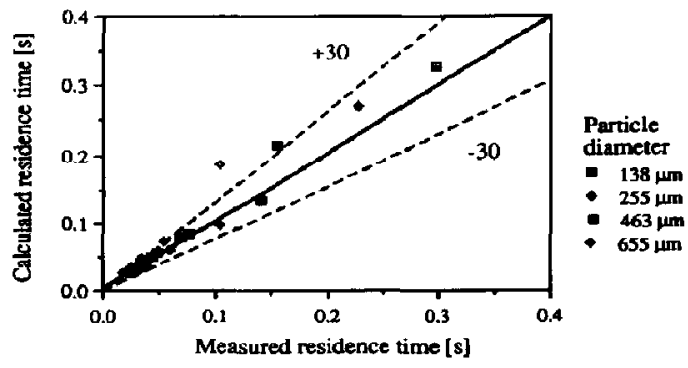

Fig. 27. Parity plot showing the calculated particle residence time vs the measured residence time experimentally determined particle residence times. Only the calculated particle residence times at a cone rotational frequency of $5.7 \mathrm{~Hz}$ deviate considerably from the measurements.

\section{CONCLUSIONS}

Based on the experimental results obtained in the present study the following conclusions can be drawn:

With the use of the photographic technique reliable measurements of local particle velocities were obtained.

- Particles larger than $400 \mu \mathrm{m}$ seem to be unaffected by the viscous forces because of their mass inertia: as a consequence the residence time of such particles is hardly dependent on the particle diameter.

- The shape of the trajectories of particles larger than $400 \mu \mathrm{m}$ is independent of the particle diameter and the cone rotational speed. If the cone rotational speed is increased, the velocity of the larger particles along their trajectory also increases but the shape of the trajectory remains the same.

- If the particle diameter is smaller than $200 \mu \mathrm{m}$ the viscous forces become important compared to the mass inertia and,in this case the particle residence time is strongly dependent on the particle diameter.

Future work: Improvement of the description of the gas-phase flow behaviour can be obtained on the basis of a computational fluid dynamics model (CFD). This model should provide a detailed description of the flow pattern inside the rotating cone reactor.

Acknowledgements - This investigation was supported by the CEC-Joule programme and NOVEM. We also acknowledge R. Röeling. H. J. H. Roeterink and E. J. H. Spekenbrink for their assistance in the experimental work.

\section{NOTATION}

c speed of light, $\mathrm{m} \mathrm{s}^{-1}$

$C_{d} \quad$ drag coefficient, dimensionless

$d_{p}$ particle diameter, $\mathrm{m}$

$\mathbf{e}_{\phi}$ angular unit vector in spherical coordinates, dimensionless

$f$ electromagnetic frequency, $s^{-1}$

F force, $\mathbf{N}$

$g$ gravitational force per unit mass, $\mathrm{ms}^{-2}$

$h$ surface roughness, $m$

$I \quad$ light intensity at depth $x$ in a solids suspension, $\mathbf{W} \mathbf{m}^{-2}$

k unit directional vector, dimensionless

I unit directional vector, dimensionless

$m_{p} \quad$ particle mass, $\mathbf{k g}$

$n$ cone rotational frequency, $\mathrm{s}^{-1}$

n surface normal vector, dimensionless

$r \quad$ radius, spherical coordinates, $m$

$\boldsymbol{R}$ radius, cylindrical coordinates, $m$

$R e$ Reynolds number, dimensionless 
$u^{*} \quad$ shear velocity experienced by the cone wall, $\mathrm{ms}^{-1}$

$v$ velocity $\mathrm{ms}^{-1}$

$\mathrm{X}$ position vector, $\mathrm{m}$

$y^{+}$dimensionless distance from the cone wall, dimensionless

\section{Greek letters}

$\beta$ deflection angle, rad

$\delta$ thickness of the logarithmic layer, $m$

\& porosity, dimensionless

$\theta$ half of the cone top-angle, rad

$v \quad$ kinematic viscosity $\mathrm{m}^{2} \mathrm{~s}^{-1}$

$\rho$ density $\mathrm{kg} \mathrm{m}^{-3}$

$\tau_{p} \quad$ particle residence time, $\mathrm{s}$

$\omega$ cone angular speed, $\mathrm{rad} \mathrm{s}^{-1}$

\section{REFERENCES}

Birchenough, A. and Mason, J. S., 1976, Local particle velocity measurements with a laser anemometer in an upward flowing gas-solid suspension. Powder Technol. 14, 139.

Booth, S. W. and Ridgway, K., 1985, Powder velocities by microwave Doppler radar. Powder Technol. 45, 11.

Harris, J., 1972, Flow measurement using microwave radar techniques. Powder Technol. 6, 85.

Hartge, E. U., 1989, Experimentelle Untersuchungen zur lokalen Strömungsmechanik zirkulierender Wirbelschichten. Thesis, Universität Hamburg-Harburg, Germany.

Heertjes, P. M., Verloop, J. and Willems, R., 1970, The measurement of local mass flow rates and particle velocities in fluid-solids flow. Powder Technol. 4, 38.

Jacobs, S. J., 1989, Effective roughness length for turbulent flow over a wavy surface. J. Phys. Oceanogr. 19, 998.

Lomas, C. G., 1986, Fundamentals of Hot Wire Anemometry. Cambridge University Press, Cambridge.

Oki, K., Walawender, W. P. and Fan, L. T., 1977, The measurement of local velocity of solid particles. Powder Technol. 18, 171.

Raso, G. and Tirabassa, G., 1983, An impact probe for local analysis of gas-solid flows. Powder Technol. 34, 151.

Rosenberger, D., 1975, Technischen Anwendungen des Lasers, Chap. 3. Springer, Berlin.

Schlichting, H., 1960, Boundary Layer Theory. McGraw-Hill, New York.

Tsuji, Y., Shen, N. Y. and Morikawa, Y., 1989, Numerical simulation of gas-solid flows. I. Particle-to-wall collision. Technical Report of the Osaka University, Vol. 39, p. 233.

Wagenaar, B. M., 1993, The rotating cone reactor. Thesis, University of Twente, Enschede. 